OPEN ACCESS

Edited by:

Yi Zhao,

Institute of Computing Technology,

Chinese Academy of Sciences, China

Reviewed by:

Yuriy L. Orlov,

First Moscow State Medical

University, Russia

Runmin Wei,

University of Texas MD Anderson

Cancer Center, United States

${ }^{*}$ Correspondence:

Chunbo Zhuang

zhuangchunbo007@163.com

Specialty section:

This article was submitted to

Computational Genomics,

a section of the journal

Frontiers in Genetics

Received: 06 March 2020

Accepted: 14 September 2020

Published: 02 October 2020

Citation:

Wang $P$, Wang $X$, Zheng $L$ and

Zhuang C (2020) Gene Signatures

and Prognostic Values of $m 6 A$

Regulators in Hepatocellular

Carcinoma. Front. Genet. 11:540186.

doi: $10.3389 /$ fgene.2020.540186

\section{Gene Signatures and Prognostic Values of m6A Regulators in Hepatocellular Carcinoma}

\author{
Pei Wang ${ }^{1}$, Xiaotong Wang ${ }^{1}$, Lei Zheng ${ }^{2}$ and Chunbo Zhuang ${ }^{2 *}$ \\ 1 Department of Gastroenterology, The First Affiliated Hospital of Zhengzhou University, Zhengzhou, China, ${ }^{2}$ Department \\ of Clinical Laboratory Medicine, The First Affiliated Hospital of Zhengzhou University, Zhengzhou, China
}

N6-methyladenosine (m6A) is the most abundant mRNA modification in mammals and has been implicated in various biological processes. However, its role in hepatocellular carcinoma (HCC) remains largely unknown. In this study, we investigated the alterations of 19 main m6A regulatory genes in $\mathrm{HCC}$ and their association with clinicopathological features, including survival. The mutation, copy number variation (CNV) and clinical data of HCC patients were retrieved from The Cancer Genome Atlas (TCGA) database. We found that the $\mathrm{m} 6 \mathrm{~A}$ regulators had high frequent alterations in HCC. The alterations of m6A regulators were significantly associated with clinicopathological features as well as TP53 alteration. Patients with any mutation of the m6A regulatory genes had worse overall survival (OS) and disease free survival (DFS). Deletion of METTL16 or ALKBH5 predicted poor OS and DFS of HCC patients. Moreover, deletion of METTL16 was an independent risk factor for DFS. Low METT16 expression was association with activation of multiple metabolic pathways in HCC. Finally, by RT-PCR, we confirmed that METTL16 was downregulated in HCC, and that lower METTL16 expression was associated with poor OS. In conclusion, we reported a significant association between alterations of $\mathrm{m} 6 \mathrm{~A}$ regulators and clinicopathological features, and highlighted the importance of METTL16 among the 19 m6A regulators in HCC pathogenesis. These findings will provide new insights into the role of m6A modification in HCC.

Keywords: hepatocellular carcinoma, m6A, METTL16, TCGA, prognosis

\section{INTRODUCTION}

Hepatocellular carcinoma (HCC) is the second leading cause of cancer-related deaths worldwide, resulting in over 500,000 deaths per year (Torre et al., 2015). Despite the recent advances in surgical resection and liver transplantation, the 5-year survival rate of liver cancer patients is still less than 17\% (Miller et al., 2016). In most cases, HCC is detected at advanced stage with limited therapeutic options. Only $10-20 \%$ of the tumors are considered surgically resectable at the time of diagnosis (ElSerag, 2011). Thus, a better understanding of the molecular mechanisms underlying the initiation and progression of HCC, as well as identification of prognostic biomarkers are still needed.

N6-methyladenosine (m6A) is the most abundant chemical modification in eukaryotic mRNA (Meyer et al., 2012). The m6A modification is a reversible process regulated by the balanced activities of m6A "writer" and "eraser" proteins (writers, erasers). m6A is installed by an RNA methyltransferase complex, the m6A writer, which is consisted of methyltransferase like 3 
(METTL3), METTL14, Wilm's tumor 1-associated protein (WTAP), KIAA1429, RNA binding motif protein 15 (RBM15), and zinc finger $\mathrm{CCCH}$ domain-containing protein 13 (ZC3H13) (Liu et al., 2014). Besides, METTL16 is a newly identified RNA methyltransferase independent from the methyltransferase complex. The reversible process is conducted by the m6A erasers, including fat mass and obesity-associated protein (FTO) and alkB homolog 5 (ALKBH5) (Jia et al., 2011; Zheng et al., 2013). The m6A-modified mRNAs are specifically recognized by the m6A readers YTH domain containing proteins YTHDC1-2, YTHfamily proteins YTHDF1-3, insulin-like growth factor 2 mRNAbinding proteins IGF2BP1-3, as well as heterogeneous nuclear ribonucleoprotein HNRNPA2B1 and HNRNPC, leading to alterations of mRNA splicing, export, translation and degradation (Wang X. et al., 2014; Xiao et al., 2016; Meyer and Jaffrey, 2017). M6A modification is implicated in various biological processes including circadian rhythm (Hastings, 2013), lipid metabolism (Zhong et al., 2018), embryonic stem cell differentiation (Wang Y. et al., 2014), as well as carcinogenesis (He et al., 2018). Increasing evidence have indicated that dysregulation of m6A pathway is frequently found in malignancies such as breast cancer (Niu et al., 2019), glioblastomas (Zhang et al., 2017), acute myeloid leukemia (Paris et al., 2019), lung cancer (Liu et al., 2020), and HCC (Lan et al., 2019). It is reported that the m6A modification level of total RNA is decreased in HCC, and that METTL14 is the main factor involved in the aberrant m6A modification (Ma et al., 2017). The decreased METTL14 expression promoted tumor metastasis by reducing the m6A modification level and miR-126 expression. Another study demonstrated that the high expression of METTL3 in HCC led to increased tumor growth and m6A modification level, mediating degradation of the tumor suppressor SOCS2 through an $\mathrm{m} 6 \mathrm{~A}$ reader protein YTHDF2dependent pathway (Chen et al., 2018). Knockdown of METTL14 significantly suppressed HCC cell proliferation and migration (Chen et al., 2018). These findings underscore the complexity of m6A modification and its regulatory enzymes in HCC. The role of m6A-related factors in carcinogenesis and progression of HCC remains to be elucidated. Hence, in the present study, we analyzed the clinical and sequencing data of HCC cohort from TCGA datasets, and investigated the alteration spectrum and prognostic values of 19 main m6A regulatory genes in patients with HCC.

\section{MATERIALS AND METHODS}

\section{Ethics Statement}

We retrieved the mutation, CNV, mRNA expression, and clinical data from The Cancer Genome Atlas (TCGA) database by $\mathrm{cBioPortal}^{1}$ platform. All the data are publicly available and openaccess. We used only anonymous statistical gene expression.

\section{Data Processing}

A total of 373 HCC patients in the TCGA database with $\mathrm{CNV}$, mutation and clinical data were included in this study.

${ }^{1}$ http://www.cbioportal.org
The loss and gain levels of copy number was identified using the Genomic Identification of Significant Targets in Cancer algorithm (GISTIC). To evaluate the clinical significance of the $\mathrm{CNV}$ and mutation status, the HCC cohort was divided into two subgroups: "with CNV and/or mutation of the 19 m6A regulatory genes" and "without CNV or mutation." For separate analysis of the association between clinicopathological feature and CNV status of individual m6A regulatory gene, only the most frequent CNV type was investigated. The mRNA expression levels were calculated by RNA-Seq V2 RSEM release, which was accessed in the University of California Santa Cruz Xena data hub (UCSC $\mathrm{Xena}^{2}$ ). The immunohistochemistry (IHC) analysis of normal liver and HCC tissues were obtained from The Human Protein Atlas (HPA) database ${ }^{3}$.

\section{METTL16 mRNA Expression Analysis by Quantitative Real-Time PCR (qRT-PCR)}

A commercial HCC cDNA microarray was purchased from Outdo biotech (Shanghai, China), which contains the cDNA of 66 HCC tissues and 21 matched para-cancerous tissues. The cDNA was amplified using SYBR-Green PCR Master Mix (TAKARA) on a QuantStudio 5 system (ABI, United States) under the conditions as follows: $95^{\circ} \mathrm{C}$ for $10 \mathrm{~min}$, followed by 40 cycles of $95^{\circ} \mathrm{C}$ for $15 \mathrm{~s}$ and $60^{\circ} \mathrm{C}$ for $1 \mathrm{~min}$. The relative expression level of METTL16 mRNA was calculated by $2^{-\Delta} \Delta C t$ method using ACTB as internal control. The specific primers were as follows: METTL16-Forward: 5' - ATGTTGCGGGGTTGGTAT GA-3'; METTL16-Reverse: 5' - TGACGGAGGCAAAGCAGA TT-3'; ACTB-Forward: 5' - GAAGAGCTACGAGCTGCCTGA3'; ACTB-Reverse: 5' - CAGACAGCACTGTGTTGGCG-3'.

\section{Gene Set Enrichment Analysis (GSEA)}

The GESA-3.0.jar software was downloaded from the website of Broad Institute and ran under the support of Java 8.0 (Subramanian et al., 2005). In this study, the TCGA HCC cases were divided into two group (High and Low) according to the METTL16 expression level. Finally, 20531 genes were involved in the enrichment process. The KEGG gene sets (c2.cp.kegg.v6.2.symbols.gmt) were used in this study. Gene sets with normalized $p$-value $<0.05$ and the false discovery rate $(\mathrm{FDR})<0.25$ were considered to be significantly enriched.

\section{Statistical Analysis}

All the data were analyzed by SPSS 17.0 (IBM, Chicago, IL, United States) and GraphPad Prism 6.0 (GraphPad Software, La Jolla, CA, United States). We used chi-square or Fisher's exact test to analyze the association between the $\mathrm{CNVs}$ of $\mathrm{m} 6 \mathrm{~A}$ regulatory genes and clinicopathological characteristics. A one-way ANOVA test was used to compare the mRNA expression levels between groups of different CNV patterns. The Kaplan-Meier curve and log-rank test were performed to evaluate the prognostic values of m6A regulatory genes' alteration. Cox proportional hazard regression model was performed to determine the prognostic

\footnotetext{
${ }^{2}$ https://xena.ucsc.edu/

${ }^{3}$ https://www.proteinatlas.org
} 
values of various clinical and molecular characteristics. A $p$ value $<0.05$ was considered statistically significant.

\section{RESULTS}

\section{Mutations and CNVs of m6A Regulatory Genes in HCC Patients}

Among the 373 cases with mutation data, mutations of m6A regulatory genes were found merely in 40 independent samples (Supplementary Table S1), while the CNVs were frequently observed in 370 HCC samples with CNV data. As shown in Table 1 and Figure 1A, a total of 3099 CNV events were found in 19 m6A regulatory genes. Among them, $1727 \mathrm{CNV}$ s led to loss of copy number, and $1372 \mathrm{CNV}$ s led to gain of copy number. All the writer and eraser genes except for KIAA1429 tend to loss of copy number, while most of the copy number gain events occurred in reader genes including YTHDF1, YTHDF3, YTHDC2, IGF2BP1, IGF2BP2, IGF2BP3, and HNRNPA2B1.

In detail, the m6A writer gene KIAA1429 had the most frequent CNV events (236/370, 63.78\%), followed by METTL16 $(230 / 370,62.16 \%)$ (Figure 1B), implying the important role of

TABLE 1 | The CNV patterns of m6A regulatory genes in HCC samples $(n=370)$.

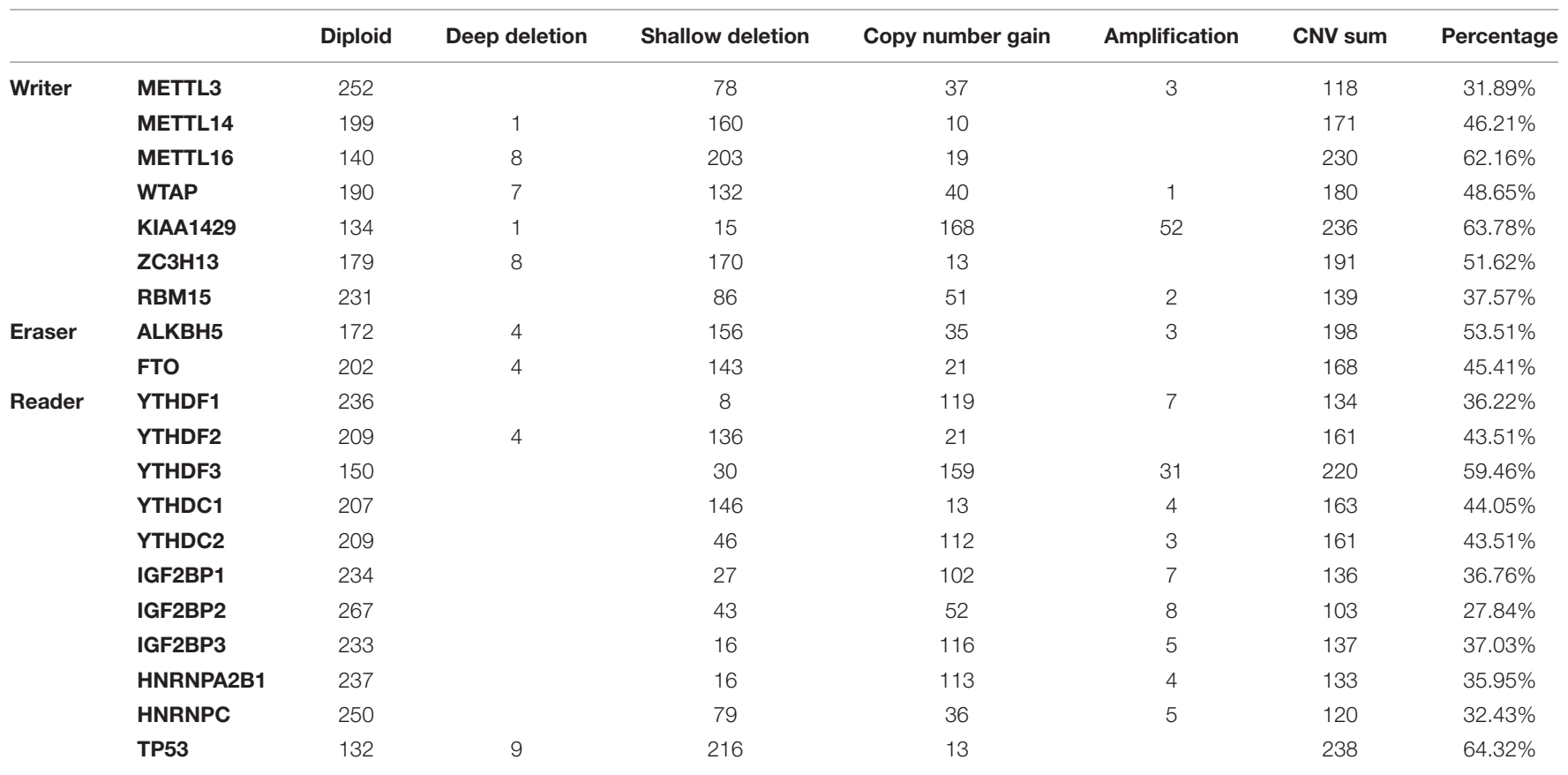

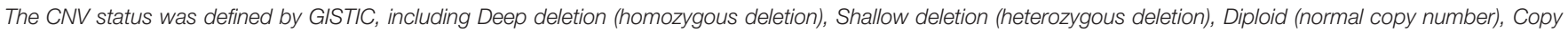
number gain (low level gain), and Amplification (high level amplification).
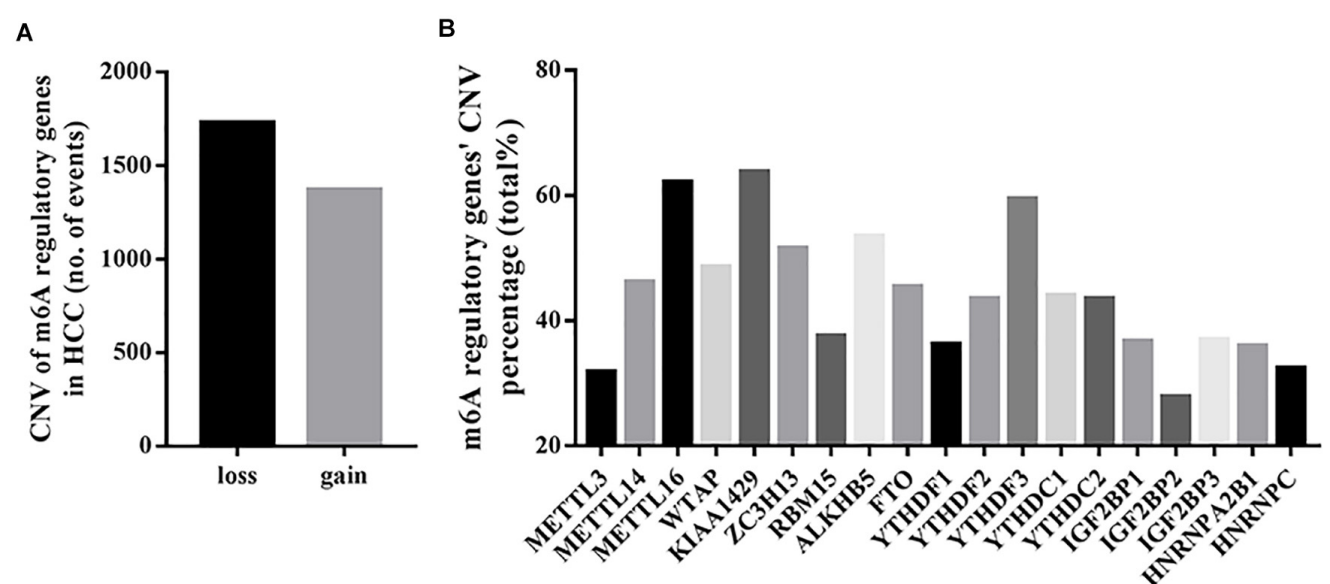

FIGURE 1 | CNVs of m6A regulatory genes in HCC. (A) Events of copy number loss or gain of m6A regulatory genes in HCC samples. (B) Percentage of HCC samples with CNVs of m6A regulatory genes according to TCGA database. 
m6A writer genes in the process of m6A modification in HCC. Furthermore, we also observed frequent mutations (115/373, $30.83 \%)$ and CNVs (238/370, 64.32\%) of TP53 (Table 1), which was in line with published literature (Nault et al., 2020).

\section{Alterations of m6A Regulatory Genes Were Associated With Clinicopathological and Molecular Features}

We evaluated the relationship between the alterations (mutations and/or CNVs) of $\mathrm{m} 6 \mathrm{~A}$ regulatory genes and the clinicopathological features of HCC patients. The result showed that, as a group, the alterations of $\mathrm{m} 6 \mathrm{~A}$ regulatory genes were only associated with gender $(p=0.0005)$ (Table 2$)$. Given the fact that TP53 play pivotal roles in the pathogenesis of HCC, we then evaluated whether the variation of m6A regulatory genes was associated with the alteration of TP53. As expected, the alterations of $\mathrm{m} 6 \mathrm{~A}$ regulatory genes were significantly associated with TP53 alteration $(p=0.0114)$ (Table 2).

We further determined whether the CNVs of individual m6A regulatory gene was associated with the clinicopathological and molecular features. The results showed that the CNVs of most m6A regulatory genes were closely related to the clinicopathological and molecular features (Supplementary
Tables S2-S20). In detail, deep or shallow deletion of METTL14, METTL16, ZC3H13, RBM15, ALKBH5, FTO, YTHDC1, and HNRNPC, and copy number gain of METTL3, KIAA1429, YTHDF1, YTHDF3, IGF2BP1, and IGF2BP2 were significantly associated with higher histological grade and/or TNM stage. Moreover, deep or shallow deletion of METTL14, METTL16, ZC3H13, ALKBH5, FTO, YTHDF2, YTHDC1, and HNRNPC, and copy number gain of IGF2BP1 and IGF2BP2 were significantly associated with the presence of TP53 alteration, which was consistent with our findings in m6A regulatory genes overall.

Next, we evaluated the effect of alterations in m6A regulatory genes on mRNA expression level. As expected, the mRNA levels were significantly associated with different $\mathrm{CNV}$ patterns. For all the m6A writers and erasers, and most of the m6A readers, deep or shallow deletions resulted in lower mRNA expression, while amplifications or copy number gains were related to higher mRNA expression (Figure 2).

\section{Association Between Alterations of $\mathrm{m} 6 \mathrm{~A}$ Regulatory Genes and Survival of HCC Patients}

We performed Kaplan-Meier analysis to investigate the impact of genetic alterations in $\mathrm{m} 6 \mathrm{~A}$ regulators on overall (OS) and

TABLE 2 | Clinical and molecular characteristics of TCGA HCC patients according to the mutation/CNV status of m6A regulatory genes.

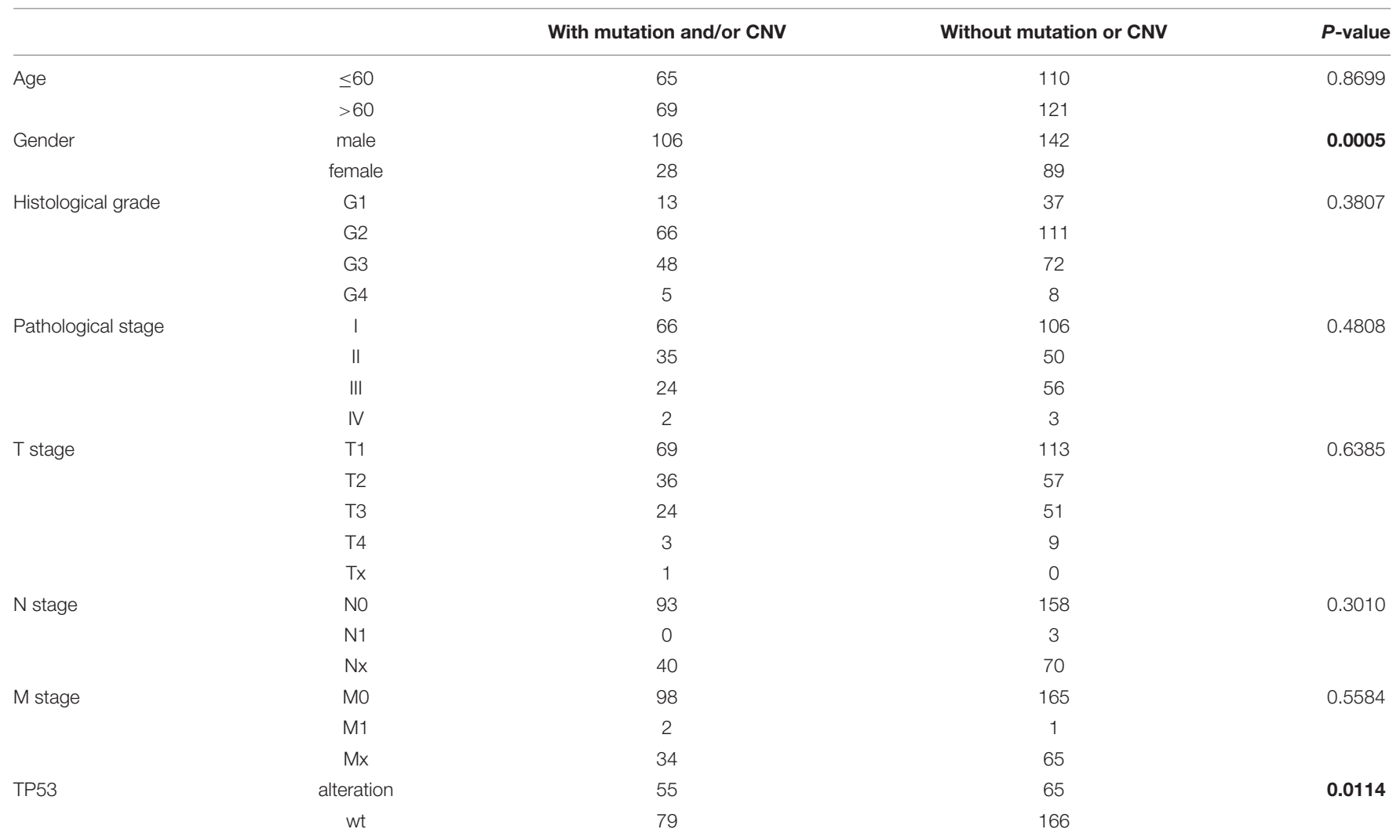

With mutation and/or CNV: cases have mutation or CNV or mutation + CNV. Without mutation or CNV: cases with neither mutation nor CNV. Ambiguous variables (Tx, $N x$, and $M x$ ) were excluded from chi-square test or Fisher's exact test. Significant $P$ values are in bold. 


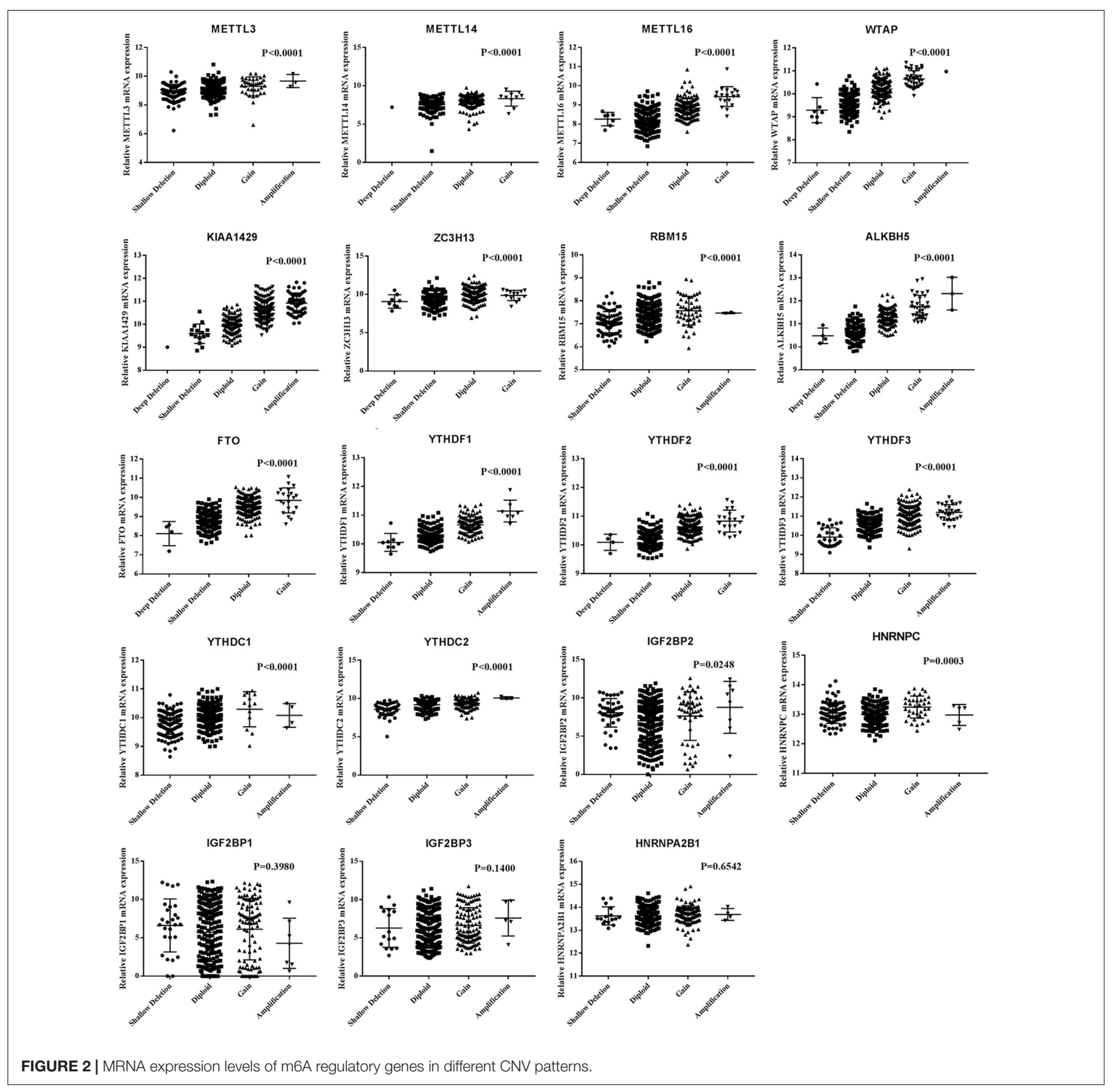

disease-free survival (DFS) in patients with HCC. As a group, patients with any mutation of the $\mathrm{m} 6 \mathrm{~A}$ regulatory genes had worse $\operatorname{OS}(P=0.0326)$ and DFS $(P=0.0213)$ (Figure 3A). However, patients with or without $\mathrm{CNVs}$ of $\mathrm{m} 6 \mathrm{~A}$ regulatory genes didn't have any correlation with OS and DFS (Figure 3B). Further separate analysis of the 19 genes revealed that patients with deletion of METTL16 or ALKBH5 had worse OS and DFS (Figures 3C,D), while no significant association between CNVs and survival was found for other m6A regulatory genes (Supplementary Figures S1, S2). To determine the prognostic values of METTL16 and ALKBH5, we performed Multivariate Cox regression analyses. The result indicated that deletion of
METTL16 was an independent risk factor of DFS for HCC patients (Table 3).

\section{The Expression of METTL16 mRNA Was Decreased in HCC and Was Associated With Poor Prognosis}

To confirm our conclusions above, we measured the mRNA level of METTL16 in 66 HCC tissues and 21 para-cancerous tissues by RT-PCR. The results showed that METTL16 mRNA was significantly downregulated in HCC tissues compared with matched para-cancerous tissues (Figure 4A, $P=0.0007$ ). 
A

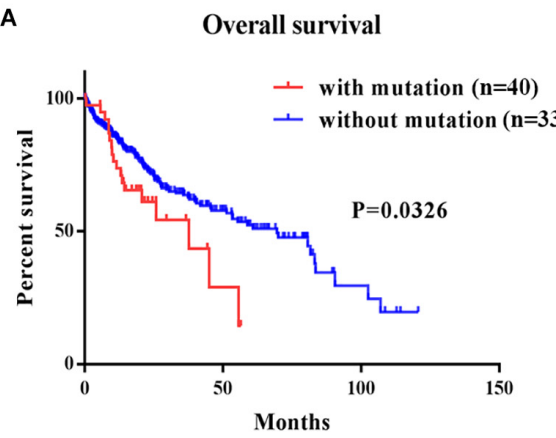

B

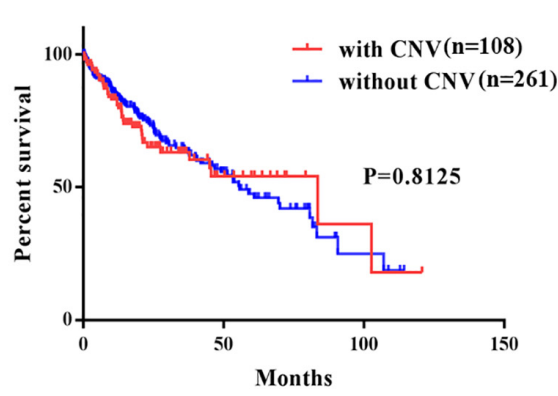

C

Overall survival-METTL16

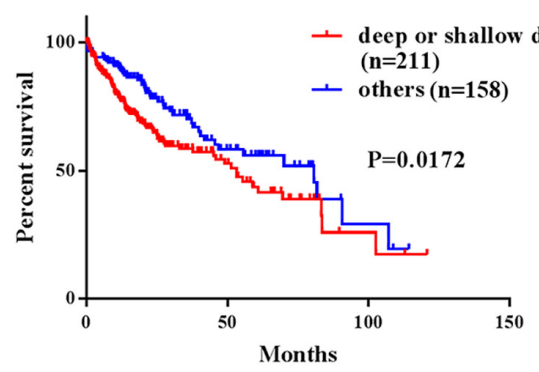

D

Overall survival-ALKBH5

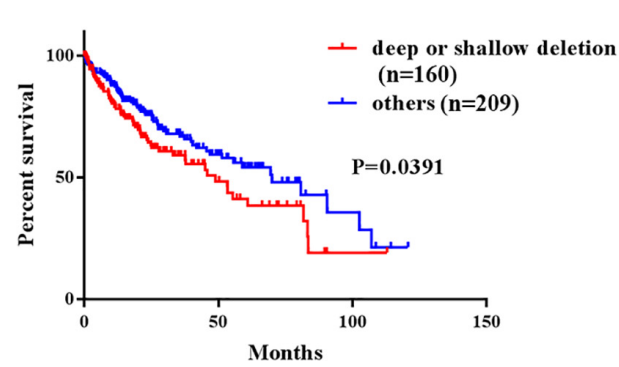

Disease free survival

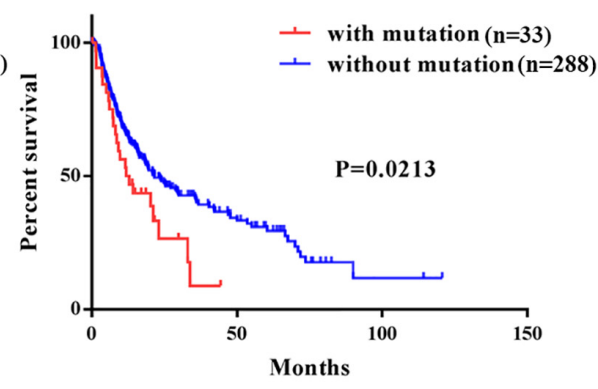

Disease free survival

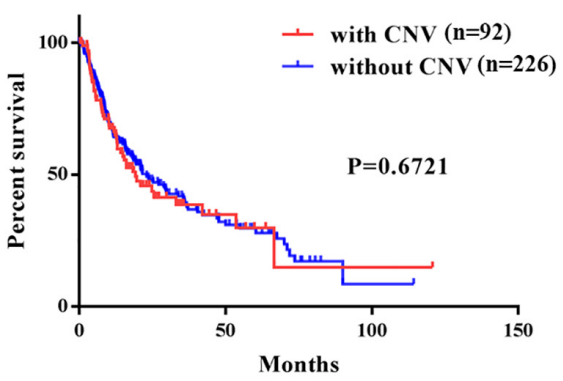

Disease free survival-METTL16

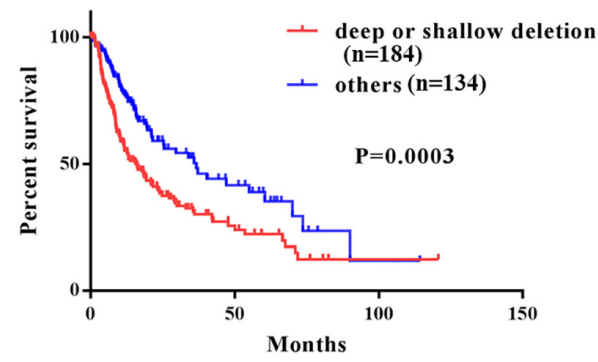

Disease free survival-ALKBH5

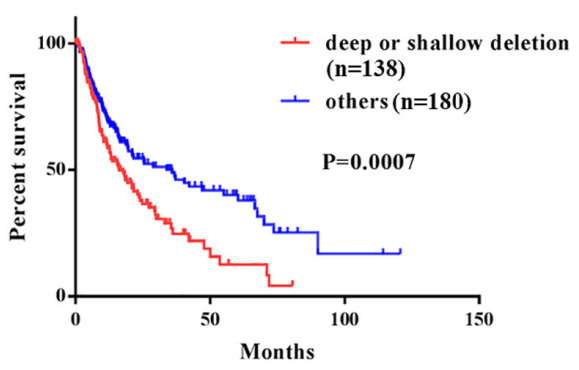

FIGURE 3 | Kaplan-Meier curves for overall and disease free survival of HCC patients in TCGA according to the presence or absence of (A) mutation of m6A regulatory genes, (B) CNV of m6A regulatory genes, (C) deep/shallow deletion of METTL16, and (D) deep/shallow deletion of ALKHB5.

Moreover, we confirmed the downregulation of METTL16 protein in HCC using the IHC staining results from the Human Protein Atlas database (Supplementary Figure S3). By searching Oncomine database, we also found a copy number loss of METTL16 gene in Guichard's HCC cohort (Supplementary
Figure S4). Then we evaluated the correlation between METTL16 mRNA expression and the clinicopathological features of HCC patients. Although no significant correlations were observed between METTL16 expression and clinicopathological features such as age, gender, pathological grade, clinical stage, and 


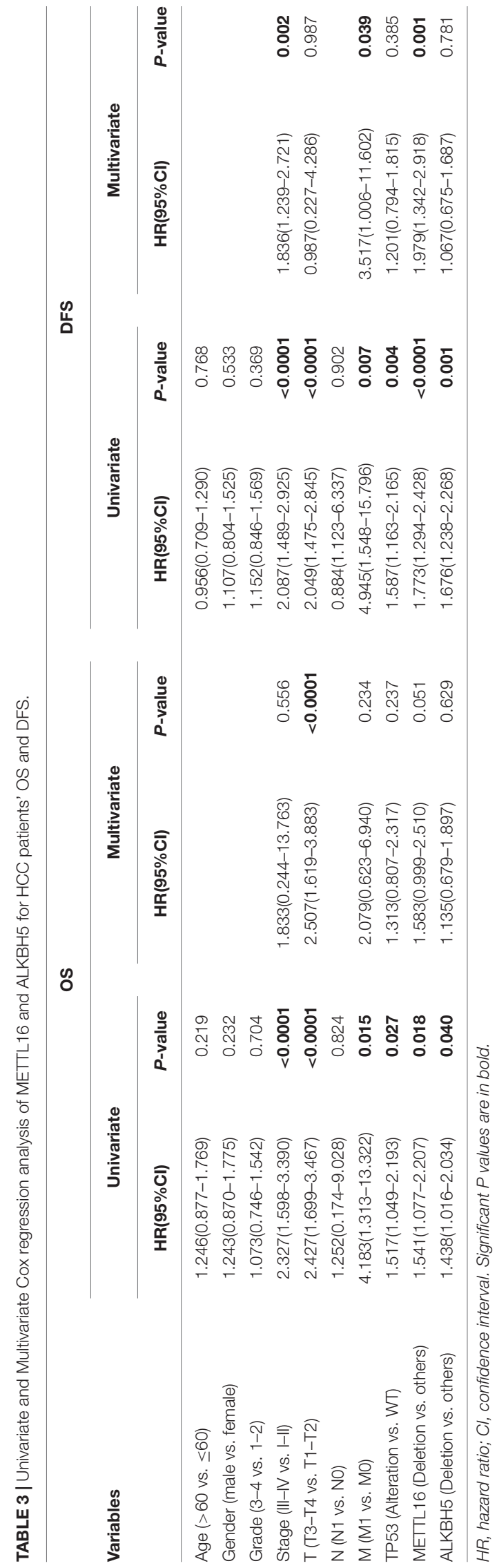

tumor size (Supplementary Table S21), we found a lower level of METTL16 in patients with stage 3/4 than that in patients with stage $1 / 2$ (Figure $4 B, P=0.0282$ ). In addition, Kaplan-Meier analysis indicated that lower METTL16 mRNA expression in HCC tissues was significantly associated with a poor OS (Figure 4C, $P=0.0367$ ). Moreover, We also investigated the association of METTL16 mRNA expression and patient survival in TCGA HCC dataset. We found a trend, though not significant, for poorer OS of patients with lower METTL16 expression levels. The mean survival time of low expression cohort and high expression cohort were 54.1 months and 81.9 months, respectively (Supplementary Figure S5). Taken together, our RT-PCR results are consistent with the findings of bioinformatic analyses.

\section{Functional Enrichment Analysis of METTL16}

Given the important role of METTL16 in m6A modification process and the interesting results we found in this study, we determined to explore the potential function of METTL16 in HCC. The gene set enrichment analysis (GSEA) were performed using data from TCGA database. The result suggested that multiple metabolic pathways including peroxisome, drug metabolism, PPAR signaling pathway, steroid hormone biosynthesis, and glycolysis gluconeogenesis, were significantly enriched in the group with low METTL16 expression (Figure 5 and Table 4). Moreover, we found that several genes related to the above pathways were significantly upregulated in HCC, which partially validated the results of GESA (Supplementary Figure S6). Further studies are needed to illustrate the functional targets of METTL16 in the pathogenesis of HCC.

\section{DISCUSSION}

Accumulating studies have reported the important role of m6A modification in tumorigenesis and progression (Wang et al., 2018). However, the alterations and biological role of m6A modification remain elusive in HCC. In this study, we analyzed the alterations of m6A regulatory genes in HCC, and found that the frequency of the alterations was much higher than that reported in acute myeloid leukemia (AML) (Kwok et al., 2017) and clear cell renal cell carcinoma (ccRCC) (Zhou et al., 2019), implying that dysregulation of m6A might play a more important role in HCC tumorigenesis. Among the 19 m6A regulatory genes, KIAA1429 and METTL16 had the most frequent $\mathrm{CNV}$ events, indicating the critical role of m6A writer genes in m6A modification of HCC. Moreover, we found that the alterations of KIAA1429 and YTHDF1 tend to gain of copy number, while METTL14 and YTHDF2 tend to loss of copy number. These findings were consistent with previous studies, which reported the upregulation of KIAA1429 (Lan et al., 2019) and YTHDF1 (Zhao et al., 2018), and downregulation of METTL14 (Ma et al., 2017) and YTHDF2 (Hou et al., 2019) in HCC.

Previous studies have suggested that the risk of developing HCC in males is higher compared with that in females 
A

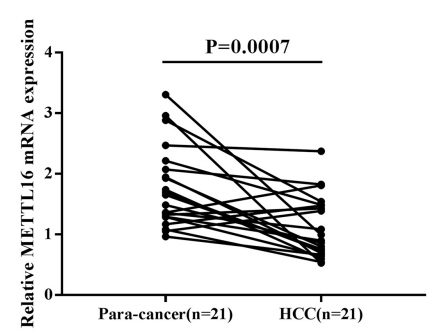

B

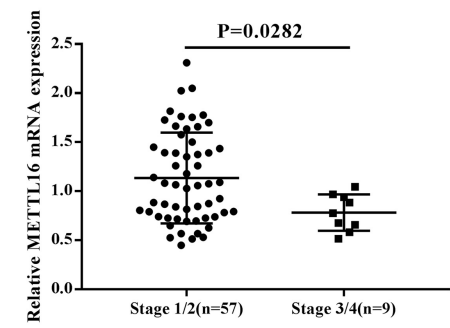

C

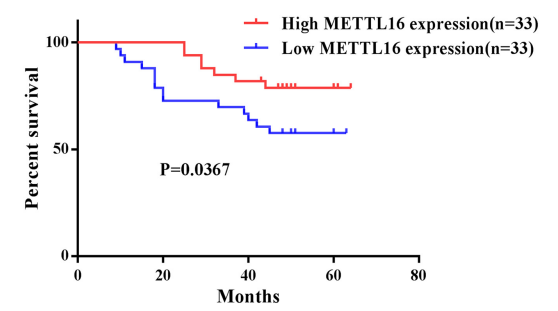

FIGURE 4 | METTL16 was downregulated in HCC and was associated with poor prognosis. (A) The mRNA level of METTL16 in 21 pairs of HCC tissues and their adjacent para-cancerous tissues. (B) The mRNA level of METTL16 in HCC patients with different clinical stage. (C) Kaplan-Meier curve for overall survival of HCC patients according to the high or low level of METTL16.

A

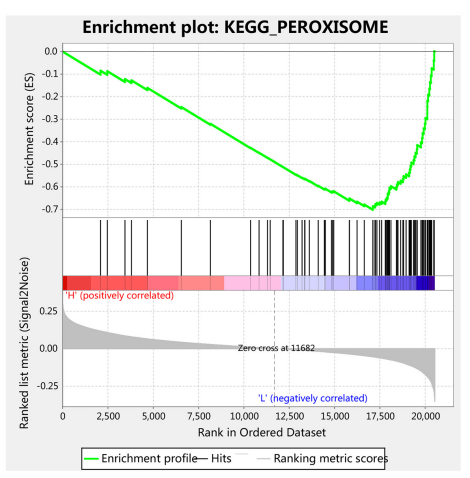

D

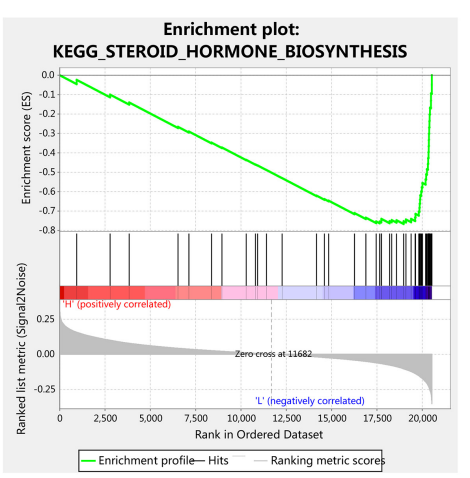

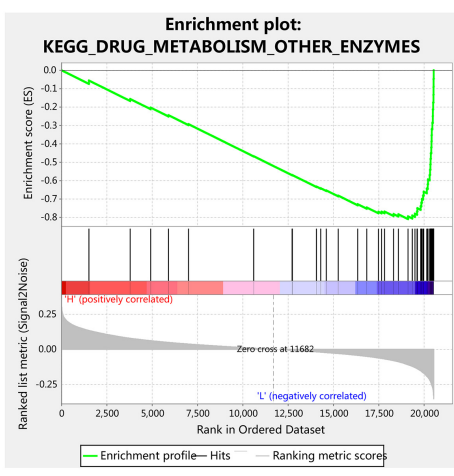

E

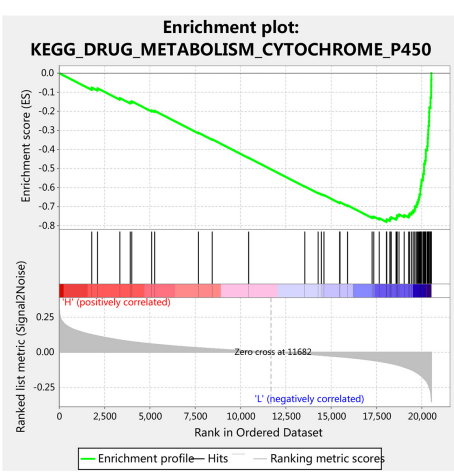

C

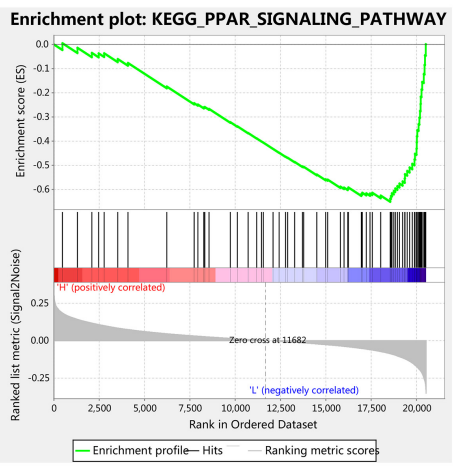

$\mathbf{F}$

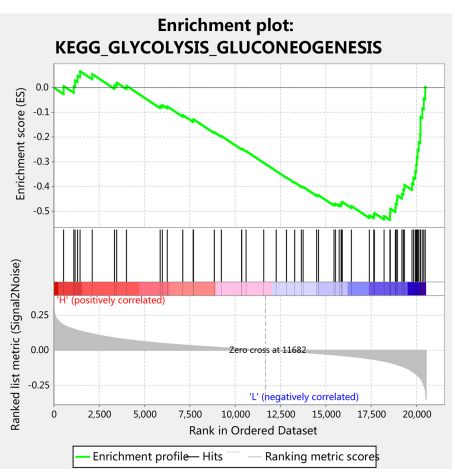

FIGURE 5 | GSEA analysis indicated that low expression of METTL16 was significantly correlated with (A) peroxisome, (B) drug metabolism other enzymes, (C) PPAR signaling pathway, (D) steroid hormone biosynthesis, (E) drug metabolism cytochrome p450, and (F) glycolysis gluconeogenesis pathways in TCGA HCC dataset. The ES indicates the degree to which the specified gene sets are overrepresented at the top/bottom of a ranked list of total protein-coding genes. For each enrichment plot, top portion: running ES for the specified gene set; middle portion: where the member of gene set appears in the ranked list of all protein-coding genes; bottom portion: value of the ranking metric as a measurement of gene's correlation with METTL16.

(Yeh and Chen, 2010; Liu et al., 2019). Irrespective of the etiology, the morbidity of liver cancer in males is $2-4$-fold higher compared with that in females (El-Serag, 2011). In this study, we found that the mutation or CNV events of m6A related genes were more common in male than that in female patients with HCC, suggesting a possible role for $\mathrm{m} 6 \mathrm{~A}$ modification in sex disparity. Moreover, we found that, for most of the m6A regulatory genes, their CNVs were significantly associated with histological grade or TNM stage, prompting that dysregulation of m6A methylation were closely correlated with HCC development. In addition, we observed the alteration of m6A regulatory genes was significantly associated with the alteration of TP53, an important tumor suppressor genes in HCC. It has been reported that silencing METTL3 in a HCC cell line HepG2 affects the expression and alternative splicing pattern of more than 20 genes involved in the TP53 signaling 
TABLE 4 | Gene sets enrichment of low METTL16 mRNA expression level in the TCGA HCC dataset.

\begin{tabular}{|c|c|c|c|c|}
\hline KEGG Pathways & ES & NES & Nominal $P$-value & FDR $q$-value \\
\hline KEGG_PEROXISOME & -0.70 & -2.04 & $<0.001$ & 0.044 \\
\hline KEGG_DRUG_METABOLISM_OTHER_ENZYMES & -0.81 & -1.86 & $<0.001$ & 0.062 \\
\hline KEGG_PPAR_SIGNALING_PATHWAY & -0.65 & -1.85 & 0.002 & 0.055 \\
\hline KEGG_STEROID_HORMONE_BIOSYNTHESIS & -0.77 & -1.73 & 0.004 & 0.110 \\
\hline KEGG_DRUG_METABOLISM_CYTOCHROME_P450 & -0.78 & -1.72 & $<0.001$ & 0.087 \\
\hline KEGG_GLYCOLYSIS_GLUCONEOGENESIS & -0.54 & -1.70 & 0.002 & 0.076 \\
\hline
\end{tabular}

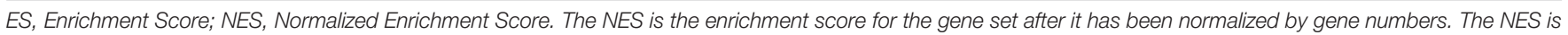
calculated by dividing the actual ES score by the mean ES of all random combinations of gene sets.

pathway (Dominissini et al., 2012). Thus, genetic alterations of m6A regulators may cooperate with TP53 signaling pathway in the pathogenesis of HCC.

We also evaluated the effect of m6A regulators' alterations on the survival of HCC patients. We found that patients with any mutation of $\mathrm{m} 6 \mathrm{~A}$ regulatory genes had a worse OS and DFS. Further separate analysis showed that only METTL16 and ALKBH5 among the 19 regulators were associated with survival of HCC patients. Moreover, the result from the Multivariate Cox regression analysis indicated that copy number loss of METTL16 was an independent risk factor for DFS. It has been reported that several $\mathrm{m} 6 \mathrm{~A}$ regulators including METTL3 (Chen et al., 2018), METTL14 (Ma et al., 2017), WTAP (Chen et al., 2019), KIAA1429 (Lan et al., 2019), and YTHDF2 (Hou et al., 2019), play critical roles in the carcinogenesis and progression of HCC. Here, we report for the first time that the alterations of METTL16 and ALKBH5 are closely associated with worse clinical characteristics including survival, and loss of METTL16 is an independent risk factor of DFS, suggesting their potential roles in HCC carcinogenesis and progression.

Recently, the mRNA methylation complex containing METTL3, METTL14, and WTAP has been the subject of intense study in human cancers (He et al., 2019). METTL16 is an independent RNA m6A methyltransferase that can methylate both coding and non-coding RNA, but its biological role is rarely studied. Although many potential RNA targets of METTL16 have been identified by high throughput method, only a few, including U6 snRNA (Warda et al., 2017), Long non-coding RNA MALAT1 (Brown et al., 2016), and mRNA MAT2A (Pendleton et al., 2017) have been verified. The MAT2A gene encodes a methionine adenosyltransferase that catalyzes the production of S-adenosylmethionine, a key substrate required for RNA methylation. It is reported that METTL16 could induce splicing of MAT2A mRNA, which increases the stability and translation of MAT2A mRNA (Pendleton et al., 2017). Thus, loss of METTL16 may result in a transcriptome-wide loss of m6A methylation by modulating S-adenosylmethionine production (Koh et al., 2019). In this study, we observed a copy number loss with down-regulation of METTL16, which might explain the previous findings that the m6A level of total RNA was decreased in HCC (Ma et al., 2017; Hou et al., 2019).

Metabolic reprogramming has been recognized as a hallmark of cancer (Pavlova and Thompson, 2016). The cancer cells reprogram their metabolism to support their rapid proliferation.
A previous TCGA analysis highlighted the critical role of metabolic reprogramming in the progression of HCC (Cancer Genome Atlas Research Network, 2017). In the present study, to explore the potential biological role of METTL16 in HCC, we performed GSEA analysis in the TCGA cohort and found that low METTL16 level was associated with the activation of multiple metabolic pathways, implying a potential role of METTL16 in metabolic reprogramming.

In conclusion, our present study determined the alterations of $19 \mathrm{~m} 6 \mathrm{~A}$ regulatory genes in $\mathrm{HCC}$ and their association with clinicopathological features including survival. We found significant associations between the genetic alterations and clinicopathological features as well as TP53 alteration. Notably, we found that loss of METTL16 predicted a worse survival and was an independent risk factor of DFS for HCC patients. Our study highlight METTL16 as a potential prognostic biomarker as well as potential target for HCC treatment. These findings provide a new insight into the underlying mechanism of m6A modification in tumorigenesis and development of HCC.

\section{DATA AVAILABILITY STATEMENT}

The datasets generated for this study can be found in The Cancer Genome Atlas (https://portal.gdc.cancer.gov/).

\section{ETHICS STATEMENT}

Written informed consent was obtained from the individual(s) for the publication of any potentially identifiable images or data included in this article.

\section{AUTHOR CONTRIBUTIONS}

PW and CZ conceived and designed the study and wrote the initial draft of the manuscript. PW, XW, and LZ collected and analyzed the data. All authors contributed to the article and approved the submitted version.

\section{FUNDING}

This study was supported by the National Natural Science Foundation of China (Nos. 81700514 and 81900515). 


\section{ACKNOWLEDGMENTS}

We acknowledge the TCGA database and cBioPortal platform for the clinicopathological and genetic alteration data.

\section{REFERENCES}

Brown, J. A., Kinzig, C. G., DeGregorio, S. J., and Steitz, J. A. (2016). Methyltransferase-like protein 16 binds the $3^{\prime}$-terminal triple helix of MALAT1 long noncoding RNA. Proc. Natl. Acad. Sci. U.S.A. 113, 14013-14018. doi: 10.1073/pnas.1614759113

Cancer Genome Atlas Research Network (2017). Electronic address wbe, cancer genome atlas Research N. Comprehensive and integrative genomic characterization of hepatocellular carcinoma. Cell 169, 1327-1341.e23. doi: 10. 1016/j.cell.2017.05.046

Chen, M., Wei, L., Law, C. T., Tsang, F. H., Shen, J., Cheng, C. L., et al. (2018). RNA N6-methyladenosine methyltransferase-like 3 promotes liver cancer progression through YTHDF2-dependent posttranscriptional silencing of SOCS2. Hepatology 67, 2254-2270. doi: 10.1002/hep.29683

Chen, Y., Peng, C., Chen, J., Chen, D., Yang, B., He, B., et al. (2019). WTAP facilitates progression of hepatocellular carcinoma via m6A-HuR-dependent epigenetic silencing of ETS1. Mol. Cancer 18:127. doi: 10.1186/s12943-0191053-8

Dominissini, D., Moshitch-Moshkovitz, S., Schwartz, S., Salmon-Divon, M., Ungar, L., Osenberg, S., et al. (2012). Topology of the human and mouse m6A RNA methylomes revealed by m6A-seq. Nature 485, 201-206. doi: 10.1038/ nature11112

El-Serag, H. B. (2011). Hepatocellular carcinoma. N. Engl. J. Med. 365, 1118-1127. doi: 10.1056/NEJMra1001683

Hastings, M. H. (2013). m(6)A mRNA methylation: a new circadian pacesetter. Cell 155, 740-741. doi: 10.1016/j.cell.2013.10.028

He, L., Li, H., Wu, A., Peng, Y., Shu, G., and Yin, G. (2019). Functions of N6methyladenosine and its role in cancer. Mol. Cancer 18:176. doi: 10.1186/ s12943-019-1109-9

He, L., Li, J., Wang, X., Ying, Y., Xie, H., Yan, H., et al. (2018). The dual role of N6methyladenosine modification of RNAs is involved in human cancers. J. Cell Mol. Med. 22, 4630-4639. doi: 10.1111/jcmm.13804

Hou, J., Zhang, H., Liu, J., Zhao, Z., Wang, J., Lu, Z., et al. (2019). YTHDF2 reduction fuels inflammation and vascular abnormalization in hepatocellular carcinoma. Mol. Cancer 18:163. doi: 10.1186/s12943-019$1082-3$

Jia, G., Fu, Y., Zhao, X., Dai, Q., Zheng, G., Yang, Y., et al. (2011). N6methyladenosine in nuclear RNA is a major substrate of the obesity-associated FTO. Nat. Chem. Biol. 7, 885-887. doi: 10.1038/nchembio.687

Koh, C. W. Q., Goh, Y. T., and Goh, W. S. S. (2019). Atlas of quantitative singlebase-resolution N(6)-methyl-adenine methylomes. Nat. Commun. 10:5636. doi: 10.1038/s41467-019-13561-z

Kwok, C. T., Marshall, A. D., Rasko, J. E., and Wong, J. J. (2017). Genetic alterations of $\mathrm{m}(6) \mathrm{A}$ regulators predict poorer survival in acute myeloid leukemia. J. Hematol. Oncol. 10:39. doi: 10.1186/s13045-0170410-6

Lan, T., Li, H., Zhang, D., Xu, L., Liu, H., Hao, X., et al. (2019). KIAA1429 contributes to liver cancer progression through N6-methyladenosinedependent post-transcriptional modification of GATA3. Mol Cancer 18:186. doi: 10.1186/s12943-019-1106-z

Liu, J., Yue, Y., Han, D., Wang, X., Fu, Y., Zhang, L., et al. (2014). A METTL3-METTL14 complex mediates mammalian nuclear RNA N6adenosine methylation. Nat. Chem. Biol. 10, 93-95. doi: 10.1038/nchembio. 1432

Liu, Y., Guo, X., Zhao, M., Ao, H., Leng, X., Liu, M., et al. (2020). Contributions and prognostic values of $\mathrm{m}(6) \mathrm{A}$ RNA methylation regulators in non-small-cell lung cancer. J. Cell. Physiol. 235, 6043-6057. doi: 10.1002/jcp.29531

Liu, Z., Jiang, Y., Yuan, H., Fang, Q., Cai, N., Suo, C., et al. (2019). The trends in incidence of primary liver cancer caused by specific etiologies: results from

\section{SUPPLEMENTARY MATERIAL}

The Supplementary Material for this article can be found online at: https://www.frontiersin.org/articles/10.3389/fgene. 2020.540186/full\#supplementary-material

the global burden of disease study 2016 and implications for liver cancer prevention. J. Hepatol. 70, 674-683. doi: 10.1016/j.jhep.2018.12.001

Ma, J. Z., Yang, F., Zhou, C. C., Liu, F., Yuan, J. H., Wang, F., et al. (2017). METTL14 suppresses the metastatic potential of hepatocellular carcinoma by modulating $\mathrm{N}(6)$-methyladenosine-dependent primary MicroRNA processing. Hepatology 65, 529-543. doi: 10.1002/hep. 28885

Meyer, K. D., and Jaffrey, S. R. (2017). Rethinking m(6)A readers, writers, and erasers. Annu. Rev. Cell Dev. Biol. 33, 319-342. doi: 10.1146/annurev-cellbio100616-060758

Meyer, K. D., Saletore, Y., Zumbo, P., Elemento, O., Mason, C. E., and Jaffrey, S. R. (2012). Comprehensive analysis of mRNA methylation reveals enrichment in 3' UTRs and near stop codons. Cell 149, 1635-1646. doi: 10.1016/j.cell.2012.05. 003

Miller, K. D., Siegel, R. L., Lin, C. C., Mariotto, A. B., Kramer, J. L., Rowland, J. H., et al. (2016). Cancer treatment and survivorship statistics, 2016. CA Cancer J. Clin. 66, 271-289. doi: 10.3322/caac.21349

Nault, J. C., Martin, Y., Caruso, S., Hirsch, T. Z., Bayard, Q., Calderaro, J., et al. (2020). Clinical impact of genomic diversity from early to advanced hepatocellular carcinoma. Hepatology 71, 164-182. doi: 10.1002/hep. 30811

Niu, Y., Lin, Z., Wan, A., Chen, H., Liang, H., Sun, L., et al. (2019). RNA N6-methyladenosine demethylase FTO promotes breast tumor progression through inhibiting BNIP3. Mol. Cancer 18:46. doi: 10.1186/s12943-0191004-4

Paris, J., Morgan, M., Campos, J., Spencer, G. J., Shmakova, A., Ivanova, I., et al. (2019). Targeting the RNA m(6)A reader YTHDF2 selectively compromises cancer stem cells in acute myeloid leukemia. Cell Stem Cell 25, 137-148.e6. doi: 10.1016/j.stem.2019.03.021

Pavlova, N. N., and Thompson, C. B. (2016). The emerging hallmarks of cancer metabolism. Cell Metab. 23, 27-47. doi: 10.1016/j.cmet.2015. 12.006

Pendleton, K. E., Chen, B., Liu, K., Hunter, O. V., Xie, Y., Tu, B. P., et al. (2017). The U6 snRNA m(6)A Methyltransferase METTL16 regulates SAM synthetase intron retention. Cell 169, 824-835.e14. doi: 10.1016/j.cell.2017.05.003

Subramanian, A., Tamayo, P., Mootha, V. K., Mukherjee, S., Ebert, B. L., Gillette, M. A., et al. (2005). Gene set enrichment analysis: a knowledge-based approach for interpreting genome-wide expression profiles. Proc. Natl. Acad. Sci. U.S.A. 102, 15545-15550. doi: 10.1073/pnas.0506580102

Torre, L. A., Bray, F., Siegel, R. L., Ferlay, J., Lortet-Tieulent, J., and Jemal, A. (2015). Global cancer statistics, 2012. CA Cancer J. Clin. 65, 87-108. doi: 10.3322/caac. 21262

Wang, S., Chai, P., Jia, R., and Jia, R. (2018). Novel insights on m(6)A RNA methylation in tumorigenesis: a double-edged sword. Mol. Cancer 17:101. doi: 10.1186/s12943-018-0847-4

Wang, X., Lu, Z., Gomez, A., Hon, G. C., Yue, Y., Han, D., et al. (2014). N6methyladenosine-dependent regulation of messenger RNA stability. Nature 505, 117-120. doi: 10.1038/nature12730

Wang, Y., Li, Y., Toth, J. I., Petroski, M. D., Zhang, Z., and Zhao, J. C. (2014). N6-methyladenosine modification destabilizes developmental regulators in embryonic stem cells. Nat. Cell Biol. 16, 191-198. doi: 10.1038/ncb2902

Warda, A. S., Kretschmer, J., Hackert, P., Lenz, C., Urlaub, H., Hobartner, C., et al. (2017). Human METTL16 is a N(6)-methyladenosine (m(6)A) methyltransferase that targets pre-mRNAs and various non-coding RNAs. EMBO Rep. 18, 2004-2014. doi: 10.15252/embr.201744940

Xiao, W., Adhikari, S., Dahal, U., Chen, Y. S., Hao, Y. J., Sun, B. F., et al. (2016). Nuclear m(6)A reader YTHDC1 regulates mRNA splicing. Mol. Cell. 61, 507-519. doi: 10.1016/j.molcel.2016.01.012

Yeh, S. H., and Chen, P. J. (2010). Gender disparity of hepatocellular carcinoma: the roles of sex hormones. Oncology 78(Suppl. 1), 172-179. doi: 10.1159/000315247 
Zhang, S., Zhao, B. S., Zhou, A., Lin, K., Zheng, S., Lu, Z., et al. (2017). m(6)A Demethylase ALKBH5 maintains tumorigenicity of glioblastoma stem-like cells by sustaining FOXM1 Expression and cell proliferation program. Cancer Cell 31, 591-606.e6. doi: 10.1016/j.ccell.2017.02.013

Zhao, X., Chen, Y., Mao, Q., Jiang, X., Jiang, W., Chen, J., et al. (2018). Overexpression of YTHDF1 is associated with poor prognosis in patients with hepatocellular carcinoma. Cancer Biomark. 21, 859-868. doi: 10.3233/CBM170791

Zheng, G., Dahl, J. A., Niu, Y., Fedorcsak, P., Huang, C. M., Li, C. J., et al. (2013). ALKBH5 is a mammalian RNA demethylase that impacts RNA metabolism and mouse fertility. Mol. Cell. 49, 18-29. doi: 10.1016/j.molcel.2012. 10.015

Zhong, X., Yu, J., Frazier, K., Weng, X., Li, Y., Cham, C. M., et al. (2018). Circadian clock regulation of hepatic lipid metabolism by modulation of m(6)A mRNA methylation. Cell Rep. 25, 1816-1828.e4. doi: 10.1016/j.celrep.2018.10.068
Zhou, J., Wang, J., Hong, B., Ma, K., Xie, H., Li, L., et al. (2019). Gene signatures and prognostic values of $\mathrm{m} 6 \mathrm{~A}$ regulators in clear cell renal cell carcinoma - a retrospective study using TCGA database. Aging 11, 1633-1647. doi: 10.18632/ aging.101856

Conflict of Interest: The authors declare that the research was conducted in the absence of any commercial or financial relationships that could be construed as a potential conflict of interest.

Copyright (c) 2020 Wang, Wang, Zheng and Zhuang. This is an open-access article distributed under the terms of the Creative Commons Attribution License (CC BY).

The use, distribution or reproduction in other forums is permitted, provided the original author(s) and the copyright owner(s) are credited and that the original publication in this journal is cited, in accordance with accepted academic practice. No use, distribution or reproduction is permitted which does not comply with these terms. 